\title{
Myxedema-Associated Hypercalcemia
}

\author{
Kimberly Graham, D.O., Alana K. Fearey, D.O., and Michael E. Grant, M.D.
}

\section{Introduction}

- Hypercalcemia is a relatively common clinical problem occurring when there is increased bone resorption, excessive gastrointestinal absorption, or decreased renal excretion of calcium.

- The most common causes of hypercalcemia are primary hyperparathyroidism and malignancy, accounting for $90 \%$ of all cases.

- Ten percent of cases are caused by other conditions.

\section{Case Presentation}

- A 45-year-old female with no significant past medical history, presented to her primary care physician with fatigue, falling, decreased appetite, and weakness.

- Labs showed a serum creatinine of $4 \mathrm{mg} / \mathrm{dL}$, a serum calcium of 15 $\mathrm{mg} / \mathrm{dL}$, and a TSH level of $177 \mathrm{ml} / \mathrm{L}$. No metabolic alkalosis was present.

- She was admitted to the hospital and treated with IV fluids, calcitonin, and levothyroxine.

- Her PTH and PTH-related peptide were low; further workup was directed towards evaluation of a systemic or neoplastic process as a cause of hypercalcemia, which was causing the acute renal failure.

- CT scans of the chest, abdomen, and pelvis failed to reveal any evidence of neoplasm, lymphoma, or renal abnormalities.

- Urine and serum studies were obtained to assess for multiple myeloma, revealing a polyclonal gammopathy of uncertain significance.

- Bone marrow aspirate did not reveal evidence of malignancy.

- Her condition improved and she was able to be switched from IV to oral levothyroxine.
- Her profound hypothyroidism appears to have contributed to her hypercalcemia.

- After correcting her hypothyroidism, her hypercalcemia resolved and has not recurred.

- Her renal function also improved with correction of the hypercalcemia, with a current creatinine of 1.6.

\section{Discussion}

- This case presents an important question: Can myxedema cause hypercalcemia?

- In a study published in 1962, investigators studied the effects of hypothyroidism on calcium balance in rats, based on a patient with similar findings.

- Their observations showed that "thyroid insufficiency may be accompanied by the propensity toward the development of hypercalcemia upon ingestion of large amounts of calcium."

- Although our patient was not known to be ingesting excessive amounts of calcium, there appears to be no other explanation for her hypercalcemia and its resolution other than profound hypothyroidism and its successful treatment.

- We suggest that, although apparently rare, the differential diagnosis of hypercalcemia should include profound hypothyroidism.

\section{References}

Lowe CE, Bird ED, Thomas WC Jr. Hypercalcemia in myxedema. J Clin Endocrinol Metab 1962; 22(3):261-267.

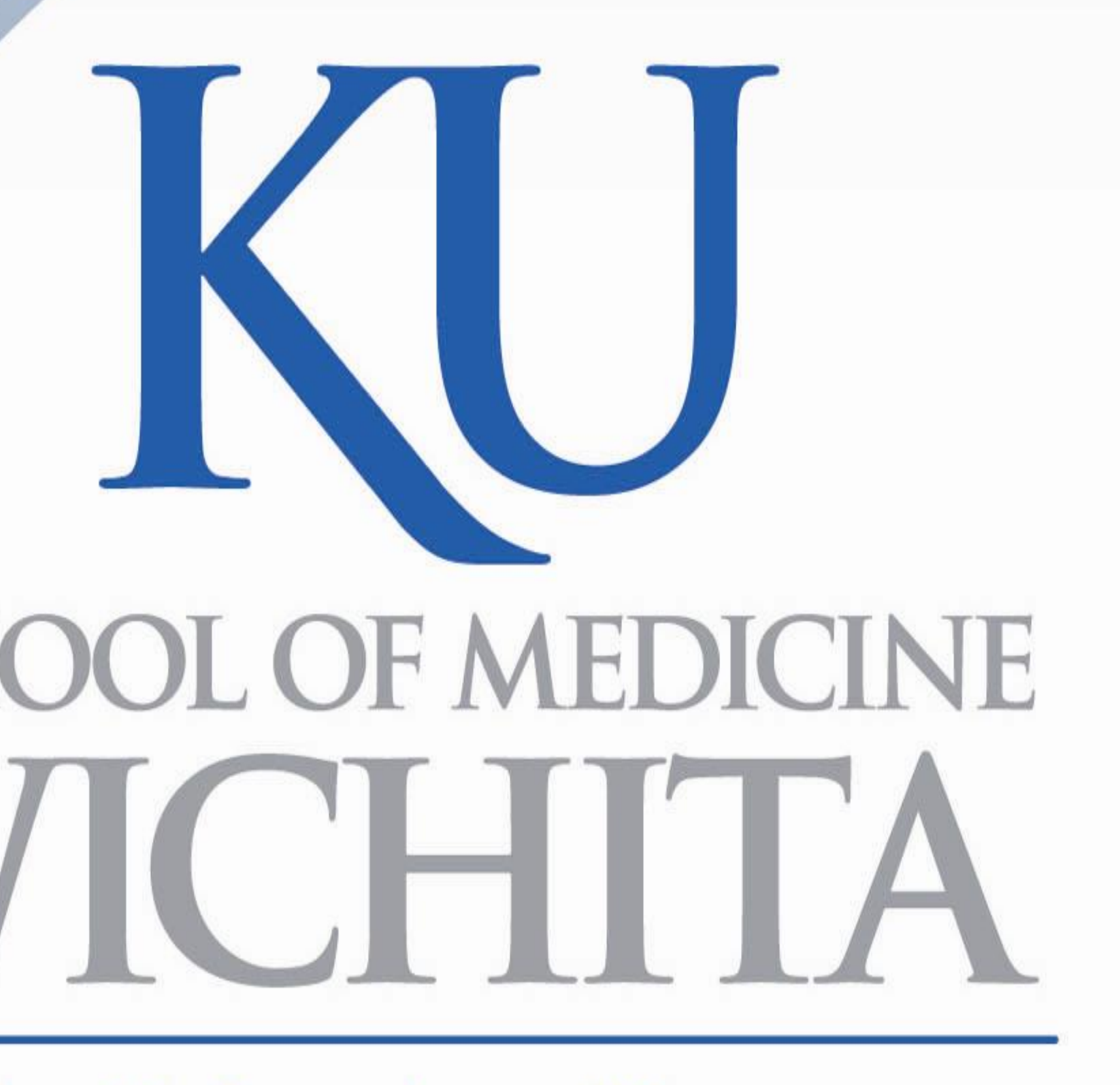

The University of Kansas 